\title{
PHILOSOPHES ET LUMIÈRES
}

\section{Peter SCHRÖDER, Naturrecht und absolutistisches Staatsrecht. Eine vergleichende Studie zu Thomas Hobbes und Christian Thomasius. Berlin, Duncker \& Humblot, 2001. 15,7 × 23,3, 241 p., bibliogr., index (Schriften zur Rechtstheorie).}

La philosophie du droit développée par Thomas Hobbes, rappelle Peter Schröder, s'articule de façon essentielle autour de deux moments, lesquels se présupposent nécessairement l'un l'autre dans le cadre d'une doctrine dont toute l'ambition a été, précisément, de proposer une structure systématique pour la philosophie politique et juridique. Ces deux moments sont, d'une part, la théorie de l'état de nature et, d'autre part, la théorie de la souveraineté absolue (p. 17-42). Or, au XVII ${ }^{\mathrm{e}}$ et au XVIII ${ }^{\mathrm{e}}$ siècle, la réception de la philosophie politique et juridique du penseur anglais n'a pas fait droit à ces deux moments de façon équivalente, déstructurant du coup l'architecture du système hobbésien. Ainsi de nombreux défenseurs de l'absolutisme ont-ils repris à leur compte la doctrine hobbésienne de la souveraineté absolue, sans suivre toutefois la théorie de l'état de nature. De leur côté, les adversaires de l'absolutisme, en France tout particulièrement, ont non seulement critiqué la doctrine de Hobbes concernant la souveraineté absolue, mais ont, eux aussi, rejeté sa doctrine concernant l'état de nature, comme le montrent nettement les pensées de Denis Diderot et de Jean-Jacques Rousseau, malgré des affinités certaines entre ces deux pensées et celle du philosophe de Malmesbury (p. 62-66).

Les théoriciens du droit et de l'État du XVII ${ }^{\mathrm{e}}$ siècle n'étaient en fait pas disposés, pour des raisons parfois fort différentes, à accepter les prémisses anthropologiques de la théorie hobbésienne de l'état de nature. C'est ce que montre très nettement la réception critique des thèses de Hobbes chez nombre de ses contemporains anglais (notamment Edward Hyde, John Eachard, Robert Filmer, James Lowde, John Shafto, John Bramhall ou encore Richard Cumberland, voir p. 43-55) ; c'est ce que montre également la réception critique des thèses de Hobbes chez les théoriciens « continentaux », allemands en particulier (Nicolaus Hieronimus Gundling, Karl Ferdinand Hommel ou bien Adam Friedrich Glafey, voir p. 56-62). Pour Schröder, Hobbes a lui-même favorisé le développement de ces critiques par une argumentation ambivalente. En effet, dans la mesure où il n'a pas seulement dégagé pour lui-même le « conflit juridique » traversant l'état de nature mais a également eu recours à des arguments de nature proprement anthropologique, les critiques se sont progressivement orientées, dans les discussions concernant le fondement du droit, vers une discussion concernant la nature des hommes. Or, en ce domaine, les arguments développés par Hobbes étaient de toute évidence hautement discutables. Mais l'essentiel, selon Schröder, est en réalité ailleurs : la réception des arguments 
hobbésiens de nature anthropologique a surtout eu pour effet de régler désormais exclusivement sur eux la pensée du droit de l'époque. Dans la philosophie de Hobbes elle-même, la question de savoir si l'homme doit être considéré comme un être altruiste ou comme un être égoïste était pourtant, fondamentalement, une question sans aucune pertinence ; ce fut cependant autour de cette question que les critiques s'embrasèrent. Pour le dire de façon tranchée, on peut avancer, selon Schröder, que les critiques anglaises contemporaines de Hobbes, malgré leurs éventuelles très grandes divergences, ont eu ceci de commun qu'elles se sont enfermées dans des formes d'argumentation traditionnelles parce qu'elles se sont concentrées, précisément, sur la signification anthropologique de la théorie hobbésienne de l'état de nature, méconnaissant du même coup sa signification juridique. Or, c'est dans cette conceptualisation proprement juridique de l'état de nature que résident pourtant l'audace, la radicalité et l'originalité de la pensée de Hobbes, laquelle fait de l'état de nature un état vide de tout droit (ein rechtloser Zustand) le droit de nature n'étant pas du droit, au sens où il ne possède aucune connotation juridique, mais un pouvoir, une force ou une liberté.

Aux yeux de Schröder, c'est à Emmanuel Kant le premier - en particulier dans le Projet de paix perpétuelle (1795), ainsi que dans la Doctrine du droit, première partie de la Métaphysique des mœurs (1796) - que l'on doit d'avoir libéré de ses éléments anthropologiques la doctrine hobbésienne de l'état de nature et de l'avoir réduite à son contenu proprement juridique, c'est-à-dire à son contenu novateur. Kant fut ainsi le premier penseur à opérer une réception positive de la théorie hobbésienne de l'état de nature, ressaisissant du même coup le caractère proprement systématique de la pensée de Hobbes (p. 219-220). Au regard de l'interprétation kantienne, la réception par Christian Thomasius de la philosophie hobbésienne du droit peut paraître, parfois, quelque peu superficielle. Seulement, soutient Schröder, lorsque l'on resitue l'interprétation de Hobbes par Thomasius dans le contexte général de la réception des thèses hobbésiennes au XVII ${ }^{\mathrm{e}}$ et $\mathrm{au} \mathrm{XVIII}^{\mathrm{e}}$ siècle, il apparaît alors très nettement que le penseur de Halle fut disposé, comme peu de théoriciens de l'époque furent prêts à le faire, à adopter la structure juridique de l'état de nature tel qu'il a été pensé par le philosophe anglais. C'est ce que montre nettement une confrontation avec la lecture de Thomas Hobbes menée par Samuel von Pufendorf (p. 66-86).

Dans le De jure naturae et gentium (1672), on se rappelle que Pufendorf développe, en s'inscrivant dans la suite directe d'Aristote et des stoïciens, l'idée d'une sociabilité naturelle de l'homme. Il en discerne deux aspects. D'une part, il y a sociabilité en ce que l'homme prend conscience de l'identité de nature qui existe entre lui-même et autrui ; c'est sur cette « conformité d'une même nature » que s'appuie la bienveillance humaine. D'autre part, il existe une moindre forme de sociabilité : l'intérêt, mode de relation distinct du premier mais qui en dérive, « car la nature en nous ordonnant d'être sociables, ne prétend pas que nous nous oubliions nous-mêmes ». Liée à l'idée de sociabilité naturelle, Pufendorf développe en outre la conception d'une loi naturelle qu'il distingue des lois révélées et des lois positives. Les lois naturelles forment, avec les lois révélées, l'ensemble des lois divines, Dieu ayant établi en tant que créateur les lois naturelles en sa sagesse 
et fait connaître les secondes en sa bonté. La théorie du droit naturel repose ainsi sur l'affirmation de l'existence d'un ordre moral universel, d'une règle de justice immuable, antérieure, indépendante et supérieure aux lois civiles. La loi naturelle est avec les lois divines une donnée première pour l'homme : produite par Dieu pour ses créatures, elle a la force et l'universalité de la droite raison, et elle possède une parfaite efficience ; enfin, «par un effet admirable de la sagesse du Créateur, les lois naturelles se trouvent tellement proportionnées à la nature humaine que leur observation est toujours avantageuse ». En ce sens, Pufendorf refuse clairement l'état de nature de Hobbes, car il unit d'emblée le respect de la loi naturelle à la nature humaine ; donnant pour essence à cette loi, fondée dans la loi divine, une parfaite rationalité, il ne saurait concevoir comme naturel un état où les hommes ne suivent pas les maximes de la raison.

Thomasius, dans les Fundamenta Juris naturae et gentium de 1705, opère ce qui doit être considéré aux yeux de Schröder comme un véritable « changement de paradigme » (Paradigmenwechsel, p. 221). Il se détourne en effet, pour penser l'état de nature, de l'idée de sociabilité naturelle et reconnaît la détermination hobbésienne de l'état de nature comme absence de droit (p. 131-161). En cela, il accomplit le chemin exactement inverse de celui de Jean de Barbeyrac : alors que Barbeyrac se détourne de Hobbes pour suivre Pufendorf, Thomasius se détourne au contraire de Pufendorf pour se rapprocher des positions hobbésiennes, jouant ainsi un rôle majeur dans la réception de la théorie hobbésienne de l'état de nature en sa dimension juridique.

Parmi les enseignements de l'ouvrage de Schröder, deux peuvent en particulier retenir l'attention. Premièrement, au regard des études hobbésiennes, les analyses de Schröder permettent de prolonger et de compléter les analyses, indispensables et non surpassées, de Samuel I. Mintz (The Hunting of Leviathan. Seventeenthcentury reactions to the materialism and moral philosophy of Thomas Hobbes, Cambridge, Cambridge University Press, 1962), puisqu'elles permettent de mieux comprendre la réception « continentale » et notamment allemande de la philosophie de Hobbes au XVII ${ }^{\mathrm{e}}$ et au XVIII ${ }^{\mathrm{e}}$ siècle. Secondement, au regard des recherches concernant la philosophie de Christian Thomasius, on apprend essentiellement deux choses : tout d'abord, que la figure de Thomas Hobbes est au moins aussi centrale que celle de John Locke pour comprendre le penseur de Halle ; ensuite, que ce dernier, en raison de sa réception novatrice de la théorie hobbésienne de l'état de nature (lue en sa dimension juridique et non pas seulement anthropologique), joue très clairement un rôle non négligeable dans la constitution du droit moderne, si du moins on accorde que celui-ci s'est formé à travers une autonomisation progressive de la sphère juridique par rapport à la sphère morale. C'est la pensée de cette autonomisation qui fait de Hobbes d'abord, de Thomasius ensuite, des figures centrales de la philosophie du droit moderne. 
Lettres d'Élie Luzac à Jean Henri Samuel Formey (1748-1770). Regard sur les coulisses de la librairie hollandaise du XVIII siècle. Éd. par Hans BoTs et Jan Schillings. Paris, Honoré Champion, 2001. 15,5 ×22,5, 416 p., index (Vie des huguenots, 15).

Voici un ensemble de deux cent treize lettres à sens unique, celles de Formey ayant été perdues. Certes dommageable, cette absence n'entame cependant en rien l'intérêt d'une publication qui, outre de précieuses informations sur l'histoire du livre et de la librairie, présente un épisode d'histoire intellectuelle.

Jean Henri Samuel Formey (1711-1797), fils de huguenot réfugié en Brandebourg après la révocation de l'édit de Nantes, fut pasteur, puis professeur de philosophie à Berlin, enfin secrétaire perpétuel de l'Académie des sciences de cette même ville. Élie Luzac (1721-1796), également descendant de huguenot, fut un libraire cultivé installé à Leyde, qui publia quelques ouvrages de son cru dans lesquels il marque son désaccord avec les idées des Lumières françaises.

En 1748, Formey, renonçant à la rédaction du Journal littéraire d'Allemagne, s'adresse au jeune Luzac pour publier un périodique analogue sous le titre de Bibliothèque impartiale. Le libraire ayant chanté les louanges du polygraphe dans l'avertissement ajouté à son édition de la Logique des vraisemblances, la communication s'établit entre les deux hommes.

Très soutenu jusqu'en 1755 , l'échange se fait plus sporadique pendant la guerre de Sept Ans. Mais, passée cette période difficile pour le commerce de la librairie, la relation d'amitié nouée depuis une quinzaine d'années ne s'en est pas moins fort refroidie. Dans la dernière lettre qu'il envoie - datée du 3 juillet 1770 -, Luzac dresse cet amer constat : «Si le gout de notre siècle eût plus répondu au notre ou si le notre eût été plus conforme au ton regnant dans la République des Lettres françoises, notre correspondance n'auroit peut-être pas tarie. Je ne me suis pas bien trouvé de vos derniers ouvrages : vous avez pris des relations avec d'autres libraires ; il n'en faloit pas davantage pour rendre la notre moins vive. »

Au plus amical de l'échange, Luzac donne quelques indications sur son caractère, son intimité. Il est droit, franc, libéral et sagement optimiste (lettre XVIII) : « [...] mon prémier principe est de supposer dans mon prochain les bonnes qualités et je les lui suppose jusqu'à ce qu'il me convainque du contraire et cette conviction n'est pas facile. » Sa philosophie est celle d'un sage (lettre XXIX) : « Je tâche de vivre en honnête homme, en être raisonnable et en être qui cherche le bien de la société autant qu'il peut y contribuer, voilà les principes qui me meuvent. Ma philosophie me met au-dessus d'une vaine gloire. Je vous estime et je plains un La Mettrie : j'ai pu rendre service à un homme dont je savois que je n' aurois aucun retour. » Sa profession de foi est déiste (ibid.) : «Je veux une religion, mais uniquement celle que la raison dicte et que le bon sens approuve [...] je ne connois d'autre religion que celui qui tend à nous tracer un Etre suprème tel que les principes de metaphysique nous l'exposent et à rendre l'univers une société d'amis. » Enfin, il n'hésite pas à faire discrètement allusion à ses démêlés avec son neveu Jean, ni à mentionner la mort de sa première épouse (lettre LXXV) : «Je ne me possederois 
point, si je ne me trouvai toujours une ferme resolution de me resigner à tout ce qu'il plaira à la Providence de me dispenser. »

Sa droiture et sa fidélité en amitié se révèlent pleinement au moment de l'affaire Koenig, en 1750-1755. Luzac admire le savant allemand et s'étonne de la conduite rigide de Pierre Louis Moreau de Maupertuis, alors directeur de l'Académie de Berlin. Formey, pour des raisons « diplomatiques », ayant sans doute tenté de tempérer ce jugement, Luzac n'en dévie pas (lettre LXXXII) : «Pourquoi voulez-vous que je vous cache mes sentiments ? Je ne vous blame pas de vous conserver M. M[aupertuis] pour ami, mais je ne voudrois pas vous voir si fort desapprouver ce que d'autres font, dans une cause qui est juste s'il en fut jamais. »

Luzac évoque à maintes reprises l'aspect commerçant de son métier. Le libraire doit être honnête en affaires afin de redorer le blason de sa profession dont le crédit (lettre IV) « est entièrement ruiné dans nos provinces aussi bien que le fort de notre négoce ». Entrepreneur rationnel, le libraire a une conscience nette de la division du travail entre lui et l'auteur (lettre II) : «Il n'est pas juste qu'un auteur travaille et que le libraire seul en profite, mais d'un autre côté c'est le libraire qui y hasarde le plus et un auteur n'en est que pour quelques heures perdues. » Luzac adopte cette politique éditoriale (lettre XV) : « Notre prémière règle est que les livres les plus universels nous sont les plus avantageux; c'est-à-dire que les livres nous conviennent en raison des lecteurs qu'ils peuvent attirer. » Le libraire demeure un commerçant au service du public, même si l'orthodoxie religieuse est en question (lettre XVIII) : " Tout comme un marchand de vin n'est pas tenu de refuser à un yvrogne tant que celui-ci peut se faire satisfaire ailleurs, ainsi un imprimeur n'est pas obligé de refuser un manuscrit que son refus n'empêcheroit pas de voir le jour. » Il y a, ajoute Luzac, une liaison d'effet à cause « entre la production d'un livre et l'usage que le public en fait, car cet usage dépend de ses caprices ». Tout l'art du libraire est de faire preuve d'esprit de finesse (lettre CVIII) : « [...] vous me permettrez de vous dire que le gout des lecteurs allemands ne decide pas de celui des François [...]. Rien n'est plus bizarre que le motif qui porte les hommes à l'achat des livres [...]. Il est vrai que le libraire profitant, l'auteur peut de son côté pretendre à quelque avantage, mais il est vrai que le succès d'un livre sert à dédommager sur le peu de profit d'un autre. » Le lecteur suit au fil des lettres les tractations complexes que conclut le libraire et les difficultés qu'il peut rencontrer avec des auteurs - Julien Offray de La Mettrie, par exemple - ou des collègues peu scrupuleux - c'est le cas de Johann Carl Spener, établi à Berlin. Enfin, Luzac envoie toujours, comme il se doit, à Formey un état précis des comptes de librairie concernant ses ouvrages.

Mais Luzac ne se veut pas qu'un commerçant. Lorsque s'élève une contestation touchant aux contrats, il donne une définition humoristique de la correspondance qu'il entretient (lettre XXI) : « [...] vous comme professeur de philosophie et moi en tant qu'étudiant en philosophie, nous sommes bien eloignés de vouloir trouver des hommes parfaits, ainsi qu'il n'y aura qu'à nous passer mutuellement des petits traits d'êtres imparfaits pour entretenir notre correspondance en philosophes. »

Luzac conçoit son métier comme l'exercice d'un véritable travail d'éditeur savant, en particulier de juge en matière de goût, et de rewriter. Il lit avec attention 
et bon sens (lettre XXXI) : « [...] nous ne croïons pas qu'on puisse démontrer dans ce qu' on cite de Thomas Burnet [...] qu'il ait avancé, que les "pièces détachées de la surface de la terre se soient enfoncées dans l'eau" [...]. Des glaçons amoncellés, par exemple, peut-on dire qu'ils se soient enfoncés dans l'eau ? Des esprits trop prévenus pour leurs sistèmes sont souvent susceptibles de ces sortes d'absences. » Il critique vivement les Lumières françaises (lettre CLXX) : «Je continue ma gazette littéraire. Je viens d'y attaquer la philosophie de d'Alembert. Je suis indigné de voir tant de vanité avec si peu de connoissances solides. Sommes-nous donc dans le siècle du stile et suffit-il savoir arranger des mots pour se donner les airs de maitre ?» Il désapprouve l'immoralisme des idées nouvelles (lettre CXC) : « [...] aujourd'hui la morale gemit sous la tyrannie d'une fausse philosophie qui paroit s'affermir de plus en plus. » En 1765, il compose un écrit contre Jean-Jacques Rousseau, dont le but (lettre CC) « est de faire voir qu'il raisonne mal, qu'il n'est point philosophe, qu'il ignore ce qu'il devroit savoir sur les sujets qu'il traitte »; et (lettre CCI) : «Jamais je ne sens plus d'ennui qu'en lisant [les] ecrits [de Rousseau]. »

C'est à l'égard de Formey, « son » auteur, que Luzac se montre le plus critique. Au début de l'échange, le libraire reproche à son correspondant d'être trop adepte de la philosophie de Christian Wolff, et, publiant une Bibliothèque impartiale, d'être trop «sectateur» et de ne pas justifier le titre de sa publication. Il le blâme nombre de fois d'écrire trop et mal (lettre CXLVII) : « [...] si en ami je puis vous dire quelque chose, c'est qu'il y a une extrême inégalité entre les pièces qui sortent de vos mains et que souvent on seroit tenté de croire que vous precipitez vos productions par des motifs d'interet. » La lettre CXC est une réécriture des Principes de morale pratique. En lui proposant des corrections de style - « p. 23. " pour faire connoitre à ceux qu'on eleve ” je voudrois : " pour convaincre ceux qui se laissent entrainer par ce gout general"»-, des remarques de fond - «p. 43. Plus vous caracteriserez les principes, Monsieur, plus vous satisferez, je crois, vos lecteurs »-, et des rajouts - «p. 51. En parlant des mauvaises compagnies vous dites: "il suffit qu'il depende de nous etc. ". J'ajouterois “et cela mène à ce principe de morale : evitez tout ce qui peut corrompre les bonnes mœurs" »-, Luzac essaie de pousser Formey à faire de l'excellent plutôt que seulement du bon, ou pis du médiocre. Mais la lettre CCVI démontre l'inutilité de ces conseils dont Formey on le devine à quelques allusions de son correspondant - dut prendre ombrage. Le polygraphe a préféré la quantité à la qualité, et aucun libraire ne veut plus se charger de ses ouvrages : «La quantité d'ouvrages que vous avez publiés, Monsieur, a indisposé le public contre vous », conclut Luzac.

Cependant le libraire, déplorant l'influence pernicieuse de la «nouvelle philosophie » des Lumières, parlera, dans sa dernière lettre, de Formey « comme [de] l'auteur qui s'est le plus et le mieux distingué contre les ouvrages dangereux ou licencieux dont nous sommes inondés aujourd'hui ». Ce compliment n'empêchera pas la rupture des relations entre les deux hommes, signe de la fin d'une époque, à la fois de la librairie et d'une conception du «polyhistor» à l'allemande que les Lumières avaient rendue caduque. 
Ces quelques extraits ne donnent qu'un faible écho de la richesse de cette correspondance dont l'intérêt ne se dément jamais. L'ouvrage contient une table chronologique des lettres, une bibliographie et un index des noms propres, très utiles et très complets. Il convient enfin de féliciter les éditeurs pour leur brève présentation, claire et précise, du sens de ces lettres, et surtout pour le remarquable appareil de notes de bas de pages, mine d'informations sur l'époque, ses hommes et ses ouvrages, qui favorise tout un système de renvois.

Jean-Marc RoHRBASSER

Gottfried Wilhelm LeIBNIZ, Doctrina conditionum. Texte intégral, prés., trad. et annot. par Pol Boucher. Paris, Institut Michel Villey/Diff. Librairie Duchemin, 1998. $13 \times 24,334$ p. (Thesaurus de philosophie du droit).

L'ouvrage comporte une présentation du traducteur ainsi que le texte en français et dans sa version originale en latin de la Doctrine des conditions, l'un des trois textes juridiques que Gottfried Wilhelm Leibniz consacre, dans les années 16651669, à l'étude du droit justinien. La philosophie et la logique y sont parties prenantes. En effet, adoptant une démarche radicalement différente de celle des principaux théoriciens de l'école moderne du droit naturel, Thomas Hobbes, John Locke, Grotius, Samuel von Pufendorf, Gottfried Wilhelm Leibniz ne conçoit pas le droit naturel comme une construction autonome de la raison destinée à réformer le droit positif, mais au contraire, comme l'expression même des sources et des mécanismes du droit justinien. Il s'essaie dès lors à une déduction entière et systématique du droit par un enchaînement de définitions et de soixante-dix théorèmes.

La Doctrine des conditions apparaît dans un contexte polémique où domine la question de l'origine des normes : divine ou naturelle ? L'abandon d'une référence extra-humaine peut réduire le droit et les règles morales qu'il contient aux normes instituées plus ou moins arbitrairement par le prince. D'où le double danger de l'absolutisme ou de l'égoïsme des subjectivités. Selon Leibniz, l'opposition entre droit révélé et droit naturel est un faux conflit provenant de la méconnaissance du droit justinien. Les deux options sont compatibles : la piété ne doit pas être exclue du droit naturel et l'équité n'est obtenue que par la considération du juste. Leibniz donne donc comme tâche au philosophe du droit de réunir en un tout plus cohérent les éléments de ce droit naturel éparpillés dans les lois et institutions et d'assurer son universalité sur les préceptes chrétiens d'une justice universelle. Ainsi, la Doctrine des conditions est l'illustration parfaite d'une défense de la moralisation du droit.

Une condition est un événement futur et incertain qui suspend ou annule la possession d'un droit. Elle se distingue du terme, événement futur et certain doté du même pouvoir suspensif et résolutoire. La condition juridique est, par définition, incluse dans une disposition prenant la forme d'une inférence où la transmission 
d'un droit est subordonnée à la réalisation préalable d'un événement. Une théorie logique des conditions a donc un double objet : l'étude de la validité de la disposition conditionnelle, d'une part, et l'étude des raisonnements concluant à la validité ou à l'invalidité des dispositions conditionnelles, d'autre part. Pour ce faire, Leibniz invente une méthode consistant à regrouper les lois par communauté de structures logiques et à les exprimer de manière déductive. C'est en logicien qu'il étudie le droit romain, en théoricien des inférences valides et invalides, et en systématicien. Contre Hobbes et la réduction du droit aux seuls rapports de force et de ruse, Leibniz utilise le modèle physique du calcul de la résultante de forces concourantes pour déterminer la solution d'un conflit juridique, solution qui requiert déjà la considération de l'équité et de la piété. On trouve également, dans la Doctrine des conditions, une ébauche de l'évaluation des risques et des espérances, à la base des futurs projets leibniziens de compagnies d'assurances. Le cas de la compensation d'une dette par une créance montre que le philosophe ne renie en rien sa méthode logique et combinatoire. Ce cas intéresse tous les degrés du droit conditionnel, intermédiaire entre le droit plein, auquel est attribuée la valeur 1 , et le droit nul, de valeur 0 . Il s'agit bien de l'ébauche d'une mathématisation des chances et des risques.

Leibniz montre que la solution de purs problèmes juridiques considérés comme insolubles n'est pas nécessairement donnée par la modification du droit qui les produit, mais plutôt par celle de ses présupposés logiques et philosophiques. Par exemple, dans la question de l'interprétation de la vente sous condition résolutoire, le philosophe résout la difficulté en donnant une définition probabiliste du contenu d'un droit conditionnel, en référence à l'existence des possibles. La Doctrine des conditions constitue ainsi une partie de la logique juridique traitant des propositions hypothétiques en droit. La condition est la proposition antécédente elle-même : «Si un navire vient d'Asie, je te donnerai 100 »; cette proposition même, « un navire viendra d'Asie », est la condition qui doit être remplie ou faire défaut.

Dans le premier chapitre, Leibniz pose, comme en mathématiques, des définitions d'où se déduisent des théorèmes généraux sur les propositions conditions. À leur énoncé succèdent leur démonstration et des références aux auteurs. Voici, à titre d'exemple, le premier théorème et sa démonstration : «La condition implique le conditionné. [Démonstration] Car si la condition est vraie, le conditionné est vrai par la déf. 3. Par conséquent, elle l'implique par la déf. 6. »

Les deux parties de l'étude des propositions conditions sont présentées dans le deuxième chapitre. L'une porte sur la nature de la condition, l'autre sur son effet. La nature, «c'est lorsque nous savons quand quelque chose est condition »; l'effet « concerne le droit et consiste en ce que nous sachions, une fois établi ce qu'est la condition, ce que ceci apporte en matière de droit ». Ainsi, le vrai se divise en légal et réel, et il convient de préciser les modalités sous lesquelles une condition peut demeurer implicite ou sous-entendue.

Après le troisième chapitre traitant des signes et de la copule dans les propositions conditions, avec les théorèmes concernant les modalités de la désignation, le quatrième chapitre étudie les propositions partielles relatives « à la Condition ellemême et au Conditionné lui-même soit absolument, soit comparativement ». Les 
théorèmes précisent l'interprétation de ces conditions. Ce chapitre termine la partie consacrée à l'interprétation en général.

Le cinquième chapitre en vient à « la multiplication des termes et à l'interprétation de la quantité » qui consiste « en règles générales la plupart du temps métaphysiques, simplement appliquées au droit », tandis que le sixième chapitre traite de l'interprétation particulière, de la forme et de la matière des propositions partielles. Le problème essentiel est ici celui de l'impossible et du possible dans les conditions. La plupart des théorèmes énoncés sont purement logiques : « le contraire du nécessaire est l'impossible » (théorème 37), ou « l'impossible est ce dont la circonstance est impossible » (théorème 39), ou encore « ce qui est impossible pour le moment, est possible dans l'absolu » (théorème 40).

Les septième, huitième et neuvième chapitres traitent de la matière des propositions partielles, respectivement des personnes, des choses et des circonstances de lieu et de temps. Pour la personne, « on requiert du conditionnateur que la chose conditionnée soit en son pouvoir» (théorème 44). Pour les choses introduites dans la condition ou dans le conditionné, « tout ce qui est de l'ordre du fait est inconditionnable » (théorème 51) ; ainsi, « les actes légitimes sont inconditionnables » (théorème 52), tout comme le droit public (théorème 53) et tout ce que le droit prescrit à propos de la qualité de citoyen (théorème 54). Pour les circonstances, « dans les dernières volontés, le jugement d'une personne bien précise est réputé être le jugement de l'Homme Bon » (théorème 61).

Le dixième et dernier chapitre traite de l'effet de la disposition tout entière, « autrement dit de la proposition conditionnelle qui est : le droit ». Ainsi, « une condition impossible rend le droit conditionnel nul, de manière régulière et en vertu du droit pur et simple » (théorème 63), tandis que « la condition nécessaire rend le droit pur» (théorème 64) et produit un droit conditionnel (théorème 65) qui « établit quelque chose dans l'Etre » (théorème 66). Ce droit conditionnel est estimé à partir du conditionné et de la probabilité de l'existence de la condition (théorème 67). Enfin, la présence ou non de la condition fait que la disposition est respectivement purifiée ou viciée (théorèmes 69 et 70).

Cet ouvrage, avec la précieuse introduction de Pol Boucher, est d'une grande importance pour la connaissance du Leibniz juriste, beaucoup moins répandue que celle du Leibniz philosophe, physicien ou mathématicien. Le lecteur dispose là d'un témoignage fidèle sur la formation fondamentale du philosophe de Hanovre et sur sa compétence en la matière. Il y trouvera en outre une remarquable application des vues logiques et mathématiques en germe chez l'auteur de la Théodicée, de son goût pour le système et l'organisation rigoureuse de données qui touchent à la probabilité comme à l'éthique, domaines en expansion dans cette seconde moitié du XVII ${ }^{\mathrm{e}}$ siècle. 
Jean-Marc RoHrbASSER et Jacques VÉRON, Leibniz et les raisonnements sur la vie humaine. Préf. de Marc BARBUT. Paris, Institut national d'études démographiques, $2001.16 \times 23,5$, XII-135 p., 12 ill. h.-t., fig. (Classiques de l'économie et de la population).

La collection «Classiques de l'économie et de la population » entend retracer l'histoire de ces deux disciplines. Elle permet de découvrir comment se sont formés des concepts et des outils de réflexion pour penser des objets nouveaux et quels sont les auteurs qui ont participé à leur élaboration. Leibniz a ainsi pris part à la naissance de ce qui deviendra la statistique et la démographie modernes. Il s'est notamment intéressé au taux de mortalité par tranche d'âge, afin d'en appliquer les déductions à la question de la rente viagère. Intérêt curieux et apparemment accessoire dans l'œuvre leibnizienne, mais dont cet ouvrage souligne le caractère novateur et important pour l'histoire de l'économie et de la démographie, montrant aussi par-là, s'il en était encore besoin, la curiosité universelle du philosophe de Hanovre. Après une préface de Marc Barbut qui permet de comprendre le rôle de Leibniz dans la naissance de la science démographique et statistique, Rohrbasser et Véron proposent une étude très précise de la méthode leibnizienne, insistant sur ses découvertes, comme sur ses tâtonnements (on notera par exemple le paramètre de la propension à la débauche pour expliquer la mortalité des 20-30 ans, laissant le soin à chacun de décider s'il s'agit d'une découverte ou d'un tâtonnement du point de vue scientifique). Le travail des deux auteurs permet donc de suivre les débuts d'une modélisation des mesures de la mortalité par tranche d'âge et des déductions économiques que l'on peut en faire, l'ouvrage se terminant par une bibliographie des textes leibniziens relatifs à ce thème et par la publication de l'un d'entre eux : l'Essay de quelques raisonnements nouveaux sur la vie humaine et sur le nombre des hommes. Il ressort de cette étude des textes que Leibniz mobilise le calcul de façon novatrice, proposant un modèle mathématique et probabiliste appliqué à la question de la «vie moyenne », c'est-à-dire de l'espérance de vie. Les auteurs soulignent d'ailleurs clairement l'intérêt de l'application de ce modèle à la question de la rente viagère, c'est-à-dire l'intérêt d'un lien fait entre espérance de gain et espérance de vie, ce qui fait que Leibniz peut être à bon droit considéré comme l'inventeur de la statistique inférentielle. C'est donc un pan du travail du Leibniz mathématicien qui est ici présenté, même si le Leibniz philosophe transparaît parfois au détour d'un calcul, d'un tableau ou d'une équation, ne serait-ce que parce que Dieu est toujours présent comme explication fondamentale de la vie et de la mort. Cet ouvrage concerne en fait les spécialistes de l'économie, de la statistique et de la démographie, mais aussi les historiens des sciences, tout en participant de l'immense travail d'exhumation des textes leibniziens qui est loin d'être achevé. Il montre enfin que tous les philosophes ne tombent pas dans des trous à force de regarder le ciel : comme Pascal et son carrosse à cinq sols, Leibniz s'intéresse à un problème concret et matériel, la rente viagère et les espérances de gains qu'elle permet d'envisager, avec toute la rigueur du mathématicien et du philosophe.

Marie-Frédérique PELLEGRIN 
Denis Thouard, Kant. Paris, Les Belles Lettres, 2001. 13,5 × 21, 176 p., bibliogr.

(Figures du savoir).

Le livre de Denis Thouard, sobrement intitulé Kant, est une introduction à la lecture de la philosophie critique d'Emmanuel Kant. Il présente de manière claire au lecteur non-spécialiste les grands axes du criticisme kantien, tel qu'il apparaît principalement dans la Critique de la raison pure, et tend à souligner l'intérêt permanent de cette philosophie ainsi que sa fécondité et sa postérité (chez des penseurs aussi différents que Johann Gottlieb Fichte, Friedrich Wilhelm Joseph von Schelling, Georg Wilhelm Friedrich Hegel, Friedrich Nietzsche, Martin Heidegger, Wilhelm Dilthey, Hermann Cohen, Ernst Cassirer, Hans-Georg Gadamer ou Jürgen Habermas).

L'introduction, intitulée «Le philosophe des philosophes », rappelle le rôle prégnant de Kant dans l'histoire de la philosophie en général et dans le siècle des Lumières en particulier, en dépassant le « portrait convenu » (p. 12) que la tradition fait souvent de Kant et du kantisme. Elle montre comment Kant « réinvente la philosophie » (p. 12). Kant, à l'instar de Socrate, dont il se réclame à maintes reprises (dans les Fondements de la métaphysique des mours ou la Doctrine de la vertu), refuse de réduire la philosophie à un savoir et a fortiori à un ensemble de dogmes ou d'idées. «Pour lui, la philosophie est d'abord un questionnement, un retour sur soi, ce qui suppose la force de s'arracher à la séduction des choses et des idées en cours [...]. C'est pourquoi, selon son expression bien connue, on n'apprend pas la philosophie, mais à philosopher. C'est un exercice de l'esprit et une certaine ascèse, plutôt que la possession d'un contenu» (p. 14). Pour Kant, disciple de Socrate au siècle des Lumières, l'essentiel en philosophie n'est pas tant le contenu que la méthode : comment pouvons-nous penser ce que nous pensons et connaître ce que nous connaissons ? L'important n'est pas tant le quoi ni le pourquoi, que le comment pense-t-on et connaît-on (p. 17). Mais la principale originalité du criticisme kantien est d'être à la fois une philosophie des Lumières (de la liberté, de la raison universelle, etc.) et une philosophie critique à l'égard des Lumières (p. 18).

La première partie de l'ouvrage, intitulée «La critique », resitue le criticisme dans le contexte historique, culturel et philosophique du siècle des Lumières, que Kant décrit lui-même, dans l'introduction à la Critique de la raison pure, comme « le siècle de la critique », voyant dans le XVIII ${ }^{\mathrm{e}}$ siècle à la fois le moment privilégié de la remise en question des préjugés et de toute forme de tutelle intellectuelle et morale, et le moment privilégié d'une possible critique des Lumières. Cette première partie souligne les principaux enjeux de la philosophie critique kantienne.

Premièrement, Kant partage les aspirations de ses contemporains à l'émancipation (de toute autorité ou coutume) et, en même temps, perçoit les contradictions inhérentes à l'Aufklärung. Prolongeant la thèse de Michel Foucault, dans Qu'estce que les Lumières? (cours au Collège de France de 1983), Thouard pense que Kant introduit « une nouvelle préoccupation dans la philosophie : l'interrogation sur le temps présent, le souci de l'actualité. Il avait conscience de vivre dans un moment historique ouvert [...]. Pour Kant, en effet, les Lumières ne désignent pas une période comme une autre [...], elles sont le présent même en train de se faire 
[...]. Le philosophe ne plane pas au-dessus des siècles, Kant le sait bien : il est immergé dans son temps, mais, pour autant, sans le suivre. Il l'interroge, il l'évalue. La philosophie n'est pas ordonnée au présent, mais, par sa capacité d'abstraction, de réflexion et de mise à distance, elle l'interprète et sonde ses contradictions » (p. 22-23). L'Aufklärung est pour Kant mouvement d'autolibération de la raison apprenant à penser par elle-même selon la devise des Lumières dans l'opuscule de 1784 : «sapere aude » (aie le courage de te servir de ton propre entendement).

Deuxièmement, la philosophie kantienne a aussi pour vocation d'apprendre, selon le titre d'un autre opuscule de Kant, à «s'orienter dans la pensée » au sens géographique (p. 27). Elle entend se repérer dans un espace de savoirs et dans une époque, sachant que le penseur n'est jamais seul, mais toujours pris dans un lieu, dans une histoire et en communication avec d'autres penseurs. Pour s'orienter dans la pensée, le sujet doit chercher en lui-même des points de repère stables et des principes de différenciation aptes à guider son jugement.

Troisièmement, pour ce faire, la philosophie doit commencer par critiquer « la raison comme sens commun » (p. 31), ce qui ne signifie pas condamner unilatéralement la raison ordinaire ou bon sens, mais en établir les droits, tant au plan pratique (de l'action) que théorique (de la connaissance). Le sens commun est en effet un possible garde-fou contre les excès spéculatifs de la raison, qui, victime d'une illusion ou dialectique naturelle, croit connaître ce qu'elle ne peut en fait que penser, à savoir les objets suprasensibles, tels le monde, l'âme, la liberté ou Dieu. Dans la Critique de la faculté de juger et l'Anthropologie du point de vue pragmatique, Kant énonce les trois maximes du sens commun, qui forment le socle de sa philosophie : penser par soi-même, en se mettant à la place d'autrui et de manière cohérente avec soi-même.

Quatrièmement, le «geste critique» (p. 33), à l'instar de la «révolution copernicienne » accomplie en astronomie et dont Kant se réclame dans la seconde préface de 1787 à la Critique de la raison pure, consiste à déplacer l'attention de l'objet de la connaissance aux conditions de sa constitution par le sujet transcendantal. Cette « révolution de la façon de penser» (p. 40) permet à Kant de donner une nouvelle «formulation du problème de la connaissance » (p. 43).

Thouard récapitule succinctement les éléments de la seule connaissance légitime, celle des phénomènes, en suivant les principales articulations de la Critique de la raison pure. Il examine successivement : a) l'espace et le temps (p. 52), formes $a$ priori de la sensibilité exposées dans l' «Esthétique transcendantale »; b) les catégories (p. 59) et les principes de l'entendement pur, constituant, au sein de la « Logique transcendantale », le contenu de l' « Analytique transcendantale »; c) le schématisme de l'imagination transcendantale (p. 69), qui donne «sens» et «signification» (p. 71) aux catégories de l'entendement; d) la fonction synthétique de l'entendement dans sa connaissance de la nature (que Kant définit formellement comme «l'enchaînement des phénomènes, quant à leur existence, d'après des lois ») ; e) les deux interprétations possibles de la Critique de la raison pure (p. 79) : celle de Heidegger (privilégiant l'irréductibilité de la sensibilité et soulignant le rôle de l'imagination transcendantale, racine commune des deux facultés de connaître que sont entendement et sensibilité) et celle des néo-kantiens, Hermann Cohen et 
Ernst Cassirer, qui accorde au contraire une importance décisive aux principes de l'entendement pur (notamment aux « anticipations de la perception » sur lesquelles ils fondent en partie la science physico-mathématique contemporaine); f) la distinction critique capitale entre phénomènes et choses en soi (p. 85) ainsi que le sens de la «Dialectique transcendantale» qui rend compte des errements de la métaphysique spéculative dogmatique traditionnelle et restreint négativement le champ de l' « Analytique transcendantale »; g) et enfin, le rôle de la méthode dans la Critique de la raison pure, dont la dernière partie est précisément une «Méthodologie transcendantale ».

La seconde partie de l'ouvrage, intitulée «Le sujet», souligne un autre aspect novateur du criticisme kantien, lié au décentrement critique de l'objet vers le sujet. Le criticisme pense à nouveaux frais la subjectivité en son rapport constitutif à l'objectivité. Thouard examine successivement : a) les quatre questions fondamentales de la raison pure et de la philosophie, à savoir : que puis-je savoir ? que dois-je faire ? que m'est-il permis d'espérer ? et qu'est-ce que l'homme ? qu'il récapitule synthétiquement (p. 101); b) l'invention par Emmanuel Kant, en rupture avec René Descartes et John Locke, d'une subjectivité transcendantale (p. 105) ; c) la dimension pratique (juridico-morale) du sujet raisonnable et responsable (p. 114), adossée à l'idée pratico-transcendantale de liberté, qui permet d'étudier le sujet dans son rapport à la loi morale (p. 117), à l'universel (p. 120), au sentiment paradoxal du respect, à la fois empirique et rationnel (p. 123), à autrui (p. 126), à l'autonomie morale (p. 128), et qui fait du sujet un membre de deux mondes à la fois, le monde sensible phénoménal et le monde intelligible nouménal (p. 135).

Enfin, la conclusion rappelle les principaux traits du criticisme kantien et son ancrage dans une nouvelle conception du sujet, au-delà même de la première Critique, en faisant place aux deux autres Critiques : la Critique de la raison pratique et la Critique de la faculté de juger. Le criticisme est une "pensée qui se pense elle-même jusque dans son imperfection constitutive », une "ascèse par laquelle une pensée lutte contre ses propres pièges » (p. 149), un retour à soi. Si le présent ouvrage a pour but de « retracer à grands traits l'entreprise de la critique de la connaissance » (p. 150), il rappelle qu'au-delà de la connaissance s'ouvrent les champs pratique (deuxième Critique), esthétique et téléologique (troisième Critique). Kant, en préparant la place d'une anthropologie critique, ouvre sa philosophie à la question « qu'est-ce que l'homme ? "L'ensemble des trois Critiques prépare ainsi, selon Thouard, par-delà l'anthropologie pragmatique empirique, une anthropologie philosophique rationnelle. C'est le criticisme qui «donne un sens à l'entreprise anthropologique, et non l'inverse » (p. 151). Le criticisme, effort réflexif de la raison sur elle-même dans les domaines théorique, pratique, esthétique et téléologique, permet la rencontre de la philosophie transcendantale et de l'anthropologie empirique et satisfait leurs exigences respectives d'universalité et de particularité. Le criticisme, au carrefour du transcendantal et de l'empirique, serait donc ultimement synthèse harmonieuse de philosophie et d'anthropologie, réunion de deux traditions : celle, philosophique, de l'universalité de la raison et celle, anthropologique et empirique, de la diversité indéfinie et contingente des façons d'être homme dans les circonstances singulières et concrètes de l'existence (p. 153). 
« Cet ouvrage se propose d'introduire à la cohérence mais aussi à l'actualité de la pensée de Kant, en exposant la signification de l'entreprise critique » (p. 178). Il constitue un précieux outil de lecture, simple et clair, une initiation au criticisme comme art de juger.

Mai-Claire LEQUAN

Christophe Bouton, Temps et esprit dans la philosophie de Hegel. De Francfort à Iéna. Paris, Vrin, 2000. 13,5 × 21,5, 320 p., bibliogr., index (Histoire de la philosophie).

Sous le titre Temps et esprit dans la philosophie de Hegel. De Francfort à Iéna, Christophe Bouton nous livre une recherche intéressante sur les rapports du temps et de l'esprit dans la philosophie spéculative. La perspective adoptée est génétique : il s'agit de suivre l'élaboration de la question de 1796 à 1807 en soulignant les acquis de chaque étape de la réflexion hégélienne, ainsi que les apories auxquelles elle se heurte.

La première partie de l'ouvrage interroge la genèse de la question du temps de 1796 à 1803. L'auteur montre comment la question de la temporalité s'articule à une réflexion sur la positivité ; dans la première version de la Positivité de la religion chrétienne (1796), Georg Wilhelm Friedrich Hegel semble adopter une perspective rationaliste qui marque l'opposition de la raison et de l'histoire, comprenant ainsi l'extériorisation de la raison comme un exil. La deuxième version de ce texte (1800) témoigne d'une transformation radicale de la pensée hégélienne, en ce qu'elle met en œuvre une conception dynamique de la nature humaine ; est ainsi rendue possible une nouvelle compréhension de la positivité comme modalité du passé s'opposant à l'actualité du présent, laissant la place à une contingence susceptible d'accueillir la rationalité. Ce bouleversement est dû au développement dans L'Esprit du christianisme et son destin, d'une ontologie de la vie tributaire de la pensée de Friedrich Hölderlin. Cette ontologie se développe selon les trois moments d'une unité contenant en soi la possibilité de la division, qui pour se développer doit restaurer son unité dans et par une division effective. L'étape de la division permet ainsi de repenser le concept de destin qui représente alors cette puissance hostile au cœur de la vie, armée par l'homme qui opère la scission de l'unité première. Ce destin est éprouvé dès lors comme nostalgie visant à rétablir l'unité malgré la séparation. C'est donc ce moment de la séparation qui rend possible la temporalité.

La volonté d'une réunion avec le temps, énoncée dans le Systemfragment de 1800, témoigne de l'achèvement aporétique de la période de Francfort ; la présence, dans la philosophie et la religion, de l'opposition du fini et de l'infini, marque le besoin d'une réconciliation avec le temps. Cette opposition ne peut être résorbée, ni par un idéal grec appartenant au passé, ni par la religion, ni par une réflexion philosophique incapable de penser l'unité du fini et de l'infini. Il y a donc nécessité de la réconciliation et impossibilité de la penser. 
Cette aporie ouvre le projet d'abolition du temps exprimé dans la Differenzschrift. La reprise de l'ontologie de la vie dans le langage de la philosophie de l'identité est à l'origine d'une modification de la temporalité qui passe du statut de destin hostile à celui d'une finitude qui doit être dépassée par la connaissance spéculative. La raison se voit donc attribuer par opposition à l'entendement une puissance d'unification à laquelle Hegel donne le nom de spéculation. La philosophie est donc maintenant habilitée à atteindre l'unité infinie du fini et de l'infini. L'Absolu est conçu comme devenir qui se temporalise dans les différents systèmes philosophiques, mais paradoxalement ce devenir est anhistorique. Hegel se refuse donc à penser une véritable historicité de la raison tout en maintenant l'exigence de sa réconciliation avec le temps. La raison ne cherche pas selon la mauvaise infinité de la temporalité pratique fichtéenne à réconcilier le temps et la raison dans le temps lui-même, c'est-à-dire dans l'élément même de l'opposition; au contraire, pour la raison spéculative, la réunion avec le temps est paradoxalement une suppression du temps dans la figure d'un présent atemporel qui est, pour l'auteur, un présent détemporalisé. C'est ce présent (Gegenwart) qui opère la médiation entre le temps et l'éternité. La signification paradoxale de ce présent reste pourtant inélucidée dans le cadre de la Differenzschrift.

La volonté de dépasser le temps reconduit l'opposition de la temporalité et de l'éternité, problème auquel se consacre Hegel dans Foi et savoir. Ce texte accentue encore le primat de l'éternité au travers d'une discussion de l'interprétation, par Friedrich Heinrich Jacobi, des rapports du temps et de l'éternité chez Baruch Spinoza. Le temps n'est rien en lui-même, cela ne veut pas dire qu'il n'est absolument pas, mais qu'il n'est pas absolument ; raison pour laquelle il peut disparaître dans l'éternité, comprise comme intemporalité et simultanéité absolues. Mais, si le temps n'est rien en soi, on ne peut guère comprendre comment le devenir de l'Absolu est possible. Pour Bouton, Hegel esquisse pourtant une autre voie susceptible de réorienter le problème dans la direction de l'histoire, c'est-à-dire dans la pensée d'une historicité de l'Absolu, avec l'image du vendredi saint spéculatif.

L'article sur le droit naturel substitue à l'analyse rationaliste de la temporalité une compréhension de l'histoire fondée sur la notion d'éthicité (Sittlichkeit). Dans cette perspective, la détermination de l'éthicité comme tout vivant implique une conception organique de l'histoire, équilibre précaire entre les différentes puissances éthiques en oscillation permanente entre naissance et mort. L'opposition centrale qui affecte ce tout vivant est celle d'une positivité morte face à une contingence vivante. La contingence, comme particulier relié avec le tout de l'éthicité, est la part inorganique nécessaire à toute vie. Elle permet, par opposition à la positivité, une manifestation temporelle de l'esprit dans l'histoire. La dimension destructrice de l'histoire ne provient pas simplement de la disparition du présent dans le passé, mais advient lorsque le passé se sépare de la totalité éthique et menace sa cohésion. Avec le concept de présent vivant (lebendige Gegenwart), Hegel conçoit enfin une figure de la temporalité qui n'est pas simplement finie. L'opposition de la positivité et de la contingence se peut dès lors traduire en celle du passé mort et du présent vivant. Le risque ne vient pas du passé mort en tant que tel, mais de la volonté de le lier malgré tout au présent vivant au lieu de l'abandonner. Parce qu'il saisit le 
présent en son historicité, Hegel commence à saisir le devenir de l'Absolu comme histoire de la vie éthique ; cependant, l'opposition de l'esprit et du temps se renouvelle sans cesse comme opposition entre le passé et le présent. L'esprit peut certes se purifier du passé, mais ce n'est là qu'une solution négative qui refoule le passé au lieu de le reprendre dans l'intériorité du souvenir. Qui plus est, la conception cyclique de l'histoire présentée dans cet article empêche tout enrichissement positif du présent par le passé. La négativité du temps - représentée par le passé mort - fait de l'histoire une figure de la tragédie de l'Absolu, dans laquelle l'éthicité se soumet à la puissance destinale du temps, et se trouve sans cesse obligée de se purifier du poids du passé.

La question de la réunion de l'esprit et du temps suppose encore une clarification de l'unité de l'infini et du fini, menacée par la présence négative du temps au cœur de toute vie. S'ouvre alors la partie la plus intéressante du travail de Bouton, consacrée à l'élucidation de la dialectique du temps dans les projets de système d'Iéna.

L'auteur éclaire ici parfaitement ces textes réputés pour leur difficulté et leur obscurité. Cette deuxième partie constitue l'apport incontestable de l'ouvrage à la question de la genèse de la théorie hégélienne de la temporalité. Face à la physique newtonienne qui, pour lui, présente un concept abstrait de matière, Hegel comprend celle-ci de manière dynamique ; le temps est opposé à l'espace et référé au point, permettant ainsi de penser le mouvement et introduisant une tension dynamique au cœur de la matière. La subjectivité de l'esprit est assimilée au point et le point au temps. Mens tempus est : une nouvelle manière de penser la possibilité de la réunion de l'esprit et du temps. Les fragments de 1803-1804 fondent la prééminence de l'esprit sur la nature et permettent de distinguer quatre modalités du temps hiérarchisées : le temps indéfini de l'inorganique, le temps vivant de l'animal, le temps particularisé de l'homme et celui libéré de l'esprit qui s'est rendu maître du passé par sa négation. Le concept de présent absolu - privilège de l'esprit - désigne alors le temps libéré du passé et de l'avenir, auquel s'ouvre une conscience libérée de la nostalgie du passé grec ou de l'attente chrétienne.

Une deuxième section interroge les rapports du temps et de l'infini dans le projet de système de 1804-1805. Hegel présente l'essence du temps comme négativité et infinité. Il élabore une dialectique dans laquelle le maintenant (Jetzt) exclusif est nié par l'avenir, nié à son tour par le passé. La double négation du maintenant est affirmation du présent concret comme unification dialectique du maintenant et de l'avenir, le caractère total du temps - dans la figure du jadis - dépendant uniquement de ce que le maintenant passé est encore présent comme présent du passé. Cependant, la négativité du temps s'oppose à son infinité, et cette totalité est sans cesse détotalisée. Bouton montre qu'un passage par la théorie des deux infinis exprimée dans la logique d'Iéna permet de faire le départ entre deux figures du temps : la répétition indéfinie du maintenant et le temps concret qui permet de résoudre l'aporie de la mauvaise infinité et qui fonde la possibilité de l'histoire. Dans la version de 1805-1806, la dialectique des deux infinis passe à l'arrière-plan, et Hegel opère une réhabilitation philosophique du temps ; au lieu d'opposer le temps et l'éternité, il essaie de penser leur unité dans une philosophie de l'esprit : c'est le temps luimême, dans sa figure concrète qui est la vraie figure de l'éternité. L'histoire 
vivante, engendrée par l'esprit, peut être considérée comme la réconciliation du temps et de l'esprit. Elle est le temps pensé, dépassement du temps au sein même du temps. Se pose pourtant encore la question du passage du temps naturel abstrait au temps réel concret, problème qui pour l'auteur sera celui de la Phénoménologie de l'esprit.

La troisième partie de l'ouvrage est consacrée à l'œuvre de 1807, et se veut une analyse de la certitude sensible, de la section « Religion» et du chapitre sur le savoir absolu. La première figure de l'ouvrage montre, pour l'auteur, que le dépassement de la négativité du maintenant est accompli par le langage et dans une moindre mesure par le souvenir. Suit une analyse de la temporalité représentative de la religion, temporalité en attente marquée par le maintien l'un en dehors de l'autre de chacun des moments de l'histoire divine. Enfin, Bouton reprend la thèse célèbre de Pierre-Jean Labarrière concernant la sursomption du temps et l'histoire conçue, et montre comment cette dernière se fonde sur une théorie de l'Erinnerung. On appréciera, en annexe, la reproduction de la traduction des manuscrits d'Iéna sur le temps que l'auteur avait publiée dans la revue Philosophie. On peut néanmoins se demander pourquoi la section «Esprit» de l'ouvrage n'a pas été considérée ; il nous semble qu'elle contenait des indications centrales sur le temps. Il nous paraît aussi difficile de faire jouer un rôle décisif à l'intersubjectivité dans la certitude sensible, et d'envisager cette figure comme une tentative pour la conscience de s'arracher à la négativité du maintenant plutôt que comme volonté d'éveiller la conscience à cette négativité pour la mettre en mouvement. Mais, somme toute, ces modestes réserves n'enlèvent absolument rien à la qualité de la recherche de Christophe Bouton qui fait le point sur un thème central et difficile.

David WiTTMANN

Marc CRÉPOn, Les Promesses du langage. Benjamin, Rosenzweig, Heidegger. Paris, Vrin, 2001. 13,5 × 21,5, 231 p., index (Problèmes et controverses).

L'ouvrage de Marc Crépon invite le lecteur à une redécouverte de philosophes au pluriel, pluralité évoquée par Friedrich Nietzsche dans Par-delà le bien et le mal comme une nécessité. La communauté, ainsi formée, des philosophes présentés l'est sous l'angle spécifique du rapport à la langue. Cette relation s'inscrit dans la dimension temporelle évoquée par la promesse. La promesse ou plutôt les promesses sont à comprendre comme un à venir, une possibilité pour les philosophes de se projeter dans l'histoire grâce aux expérimentations langagières. En effet, le philosophe doit adopter une stratégie face à sa propre langue, elle n'est pas un simple instrument de communication, elle est une langue particulière, particularité qui vient contredire la prétention universelle du propos philosophique. Il en découle donc possiblement l'existence d'une langue d'exception, une langue proprement philosophique, le grec ? l'allemand? Les peuples qui la parlent, la nation qui la porte pourraient puiser dans cette singularité « une mission historique universelle » 
(p. 22), attitude difficilement supportable à bien des égards et qui essentialise les communautés en laissant la place à des caractères nationaux exarcerbés. "C'est pourquoi il existe une autre sratégie - affranchie de cette superstition du génie [...] qui donne droit à la pluralité des langues, à l'exigence des passages et des traductions » (p. 23).

Les questions que posent Crépon sont nombreuses, elle nous engagent à penser l'œuvre philosophique comme une «provocation à la traduction » puisqu'elle est écrite dans une langue «qui n'appartient à personne» (p. 23). L'idée reprise au Nietzsche d'Ainsi parlait Zarathoustra permet d'envisager l'avenir. Martin Heidegger, Karl Kraus, Walter Benjamin et Franz Rosenzweig l'ont développée chacun à leur manière à travers le thème de la promesse, « ils cherchent dans le langage le lieu et la forme de cette espérance » (p. 24). Ces « signatures singulières » (p. 26) d'une autre philosophie du langage ouvrent un débat susceptible de nous éclairer sur les notions d'identité, de culture ou de cosmopolitisme très présentes dans le champ des sciences sociales et donnent l'espoir de découvrir peut-être un mode politique d'être ensemble, une hospitalité vraie.

Nous nous intéresserons, ici plus particulièrement, aux chapitres finaux du livre qui proposent diverses figures de l'autre sous les plumes de Robert Musil et Martin Heidegger (chap. VII), puis de Jacques Derrida et son « monolinguisme de l'autre » (chap. VIII), pour repenser enfin les notions de culture et de territoire à l'aide notamment du Gulliver de Jonathan Swift, voyageur échoué et emblématique (chap. IX).

Dans le chapitre VII, intitulé «La compréhension mutuelle des peuples », deux visions du problème que pose l'idée de philosophie nationale sont mises en parallèle. Pour Musil, il s'agit de déconstruire le concept de race sans réhabiliter pour autant celui de culture. Sur ce point, il met l'accent sur «l'existence d'autres formes de solidarités et de communautés transnationales » (p. 160), minant ainsi l'autorité homogène des concepts de nation, de peuple et d'État. Une réponse possible n'est plus à trouver dans une origine à redécouvrir mais dans l'appropriation d'une complexité de situations : «Où sommes-nous ? », plutôt que : «Que sommes-nous ?» Pour Heidegger, le concept de «peuple» ne doit pas être rabattu sur celui d'État ou de race. La version heideggérienne de la question «Qui sommes-nous ? renvoie au temps originel de la création des peuples, temps voilé mais que chacun doit néanmoins se réapproprier pour assumer son destin historial : «Une compréhension mutuelle des peuples n'est possible que dans la mesure où chacun reconnaît la tâche qui lui est assignée par l'histoire » (p. 162). Musil et Heidegger, malgré deux pensées éminemment différentes, ont la clairvoyance d'ouvrir la question des identités, l'offrant ainsi à la possibilité d'une promesse à venir.

Le chapitre VIII déploie également l'idée d'une déconstruction d'un mécanisme identitaire réificateur en rapportant la critique sévère de Derrida à propos de l'appropriation de la langue. Dans « le monolinguisme de l'autre », il expose l'idée d'une langue qui s'impose à nous de l'extérieur et qui n'en est pas pour autant réappropriable. La langue de l'autre n'a pas de propriété naturelle, perçue comme telle elle permet de saisir l'illusion nationaliste d'une réappropriation collective soulevant des passions destructrices : «Ce que la langue comme langue de l'autre 
(qui m'impose sa loi) révèle de la culture, ce n'est pas son caractère naturel, mais son essence coloniale » (p. 188). Derrida cherche à remédier à cette aliénation, il y parvient par l'exhortation à la création de sa langue. «C'est se donner avec elle (sa langue) le moyen de n'être jamais la colonie d'un autre» (p. 189). Cette langue pourtant ne peut exister qu'en se traduisant dans la langue de l'autre, cette « avantpremière » langue est donc une « langue promise » (p. 190). L'idée de la traduction qui renvoie à la pensée de l'enracinement chez Heidegger permet au contraire chez Derrida de dénier les frontières d'une langue ou d'une culture et d'ouvrir vers un cosmopolitisme.

C'est ce nécessaire cosmopolitisme qui est évoqué dans le chapitre suivant (chap. IX), les temps présents nous offrent une infinie circulation des personnes à travers des territoires étatiques qui toujours amplifient les lois gérant les flux de population. Comment penser alors un avenir répondant à la loi de l'hospitalité absolue ? - cette notion qu'évoque Derrida dans De l'hospitalité et qui doit rompre avec l'hospitalité de droit. Crépon illustre par l'exemple de Gulliver l'expérience des conditions de l'hospitalité. Celui-ci, étranger partout où il échoue, figure de l'autre, différent, trop petit, trop grand, ne parlant pas la langue voulue, à qui on ne donne jamais la parole, subit un rapport de force faute d'être accueilli : « Du défaut de parole donnée à la condamnation de l'hôte, la conséquence est donc implacable » (p. 201). Quand enfin il peut parler, rien ne change, le jugement de son récit par celui qui l'entend est négatif. Toutes les frontières résistent à Gulliver : « langues, culture, territoires repliés sur eux-mêmes » (p. 203). Son dernier voyage, enfin, lui permet de se traduire, d'être accueilli dans la langue. En lui accordant l'accueil de la parole, ses hôtes acceptent le risque de faire l'expérience de l'étranger. Crépon propose alors une hospitalité pensée en terme de traduction ; en invitant au sein de sa démonstration Antoine Berman et son «épreuve de l'étranger », il offre une possibilité aux chercheurs de l'interculturel de répondre peut-être à certaines difficultés. La «puissance de décentrement» (p. 213) que détient le paradigme de la traduction rassure sur la possibilité d'un avenir signifié en ces termes : «Telle qu'elle se donne, sous le masque d'une identité factice, chaque culture a en propre d'être la culture de tous et de personne. Elle est celle de personne car notre identité culturelle est toujours trop complexe pour qu' aucun "nous" puisse lui correspondre » (p. 213).

À travers ce voyage dans le rapport à la langue, dans les promesses, nous avons découvert une autre façon de penser les cultures, l'invitation à une traduction qui dénationalise, déterritorialise et nous fait accéder à une pluralité de rivages multiculturels. 